\title{
Fast Parallel Ring Recognition Algorithm in the RICH Detector of the CBM Experiment at FAIR
}

\author{
Semen Lebedev ${ }^{* a b}$, Claudia Höhne ${ }^{a}$, Ivan Kisel ${ }^{a}$, and Gennady Ososkov ${ }^{b}$ for the \\ CBM collaboration ${ }^{\dagger}$ \\ ${ }^{a}$ GSI Helmholtzzentrum für Schwerionenforschung GmbH, Darmstadt, Germany \\ ${ }^{b}$ Joint Institute for Nuclear Research, Dubna, Russia \\ E-mail: s.lebedev@gsi.de
}

\begin{abstract}
The Compressed Baryonic Matter (CBM) experiment at the future FAIR facility at Darmstadt will measure dileptons emitted from the hot and dense phase in heavy-ion collisions. In case of an electron measurement, a high purity of identified electrons is required in order to suppress the background. Electron identification in CBM will be performed by a Ring Imaging Cherenkov (RICH) detector and Transition Radiation Detectors (TRD). Very fast data reconstruction is extremely important for CBM because of the huge amount of data which has to be handled.

In this contribution, parallelized ring recognition algorithm is presented. Modern CPUs have two features, which enable parallel programming. First, the SSE technology allows using the SIMD execution model. Second, multi core CPUs enable the use of multithreading. Both features have been implemented in the ring reconstruction of the RICH detector. A considerable speed up factor from $357 \mathrm{~ms} / \mathrm{event}$ to $3 \mathrm{~ms} / \mathrm{event}$ has been achieved including preceding code optimization for Intel Xeon X5550 processors at $2.67 \mathrm{GHz}$.
\end{abstract}

13th International Workshop on Advanced Computing and Analysis Techniques in Physics Research February 22-27, 2010

Jaipur, India

\footnotetext{
* Speaker.

${ }^{\dagger}$ This work was supported by the EU Integrated Infrastructure Initiative Hadron-Physics Project (I3HP) under Contract number RII3-CT-2004-506078.
} 


\section{Introduction}

The Compressed Baryonic Matter (CBM) experiment is designed to investigate high-energy nucleus-nucleus collisions at the future international FAIR project [1]. The objective of high-energy heavy ion collision experiments is to explore the QCD phase diagram. The CBM research program includes the study of particles containing charm quarks $\left(D, J / \psi\right.$, and $\psi^{\prime}$ mesons) and low-mass vector mesons decaying into dilepton pairs ( $\rho, \omega, \phi$-mesons).

The experimental task is to identify both hadrons and leptons in a large acceptance and to detect rare probes in a heavy ion environment. The experimental challenge is to select rare events in nucleus-nucleus collisions with charged particle multiplicities of about 800 per central event at reaction rates of up to $10 \mathrm{MHz}$. In particular, such measurements require fast event reconstruction algorithms.

\section{The RICH detector}

The RICH detector in CBM will serve for electron identification from lowest momenta up to $10 \mathrm{GeV} / \mathrm{c}$ needed for the study of the dielectronic decay channel of low mass vector mesons and $J / \psi$ [2]. The current design of the RICH detector provides about 21 hits/electron ring, the ring radius of electrons is about $5 \mathrm{~cm}$. As the photodetector can only approximately be placed in the focal plane, rings are typically distorted to ellipses with about $10 \%$ difference in the length of major and minor half axis. The dimensions of the sensitive pads for the photodetector are $0.58 \mathrm{x} 0.58 \mathrm{~cm}^{2}$. The ring and hit density on the photodetector plane is non-uniform and depends on the position on the photodetector. The inner part which is closer to the beam pipe has the highest ring densities.

\section{The ring recognition algorithm}

The developed ring recognition algorithm is standalone, i.e the input data is only an array of RICH hits [3]. The algorithm consists of three steps. First, a local search of ring-candidates is performed. It is based on the Hough Transform (HT) method. The second step is a ring selection, in which mainly the quality of rings is determined using an artificial neural network (ANN). The third step is an ellipse fitting of found rings.

Ring-candidate search based on the Hough Transform method. HT is a standard method for curve recognition in digital images, e.g. for finding straight lines, circles or ellipses [4]. HT requires very large combinatorics and thus it is an intrinsically slow method. Instead of combining all possible hit triplets in the photodetector plane, we use the fact that the RICH rings have a maximum radius $R_{\max }$ because of the limiting Cherenkov angle. Hit triplets are only combined in a local area of the ring-candidate (see fig. 1, left). Hits are collected lying within a predefined region around the initial hit, which defines the preliminary position of the first ring. Then center and radius are calculated using HT equations from every triplet of selected hits and Hough histograms are filled. When the histograms are built, strong peaks in each histogram should correspond to the supposed positions of ring centers (2D histogram, see fig. 1, right) and radii (1D histogram). If the peak is higher than a prescribed cut this ring-candidate is accepted and shifted to the ring-candidate array, otherwise rejected. 


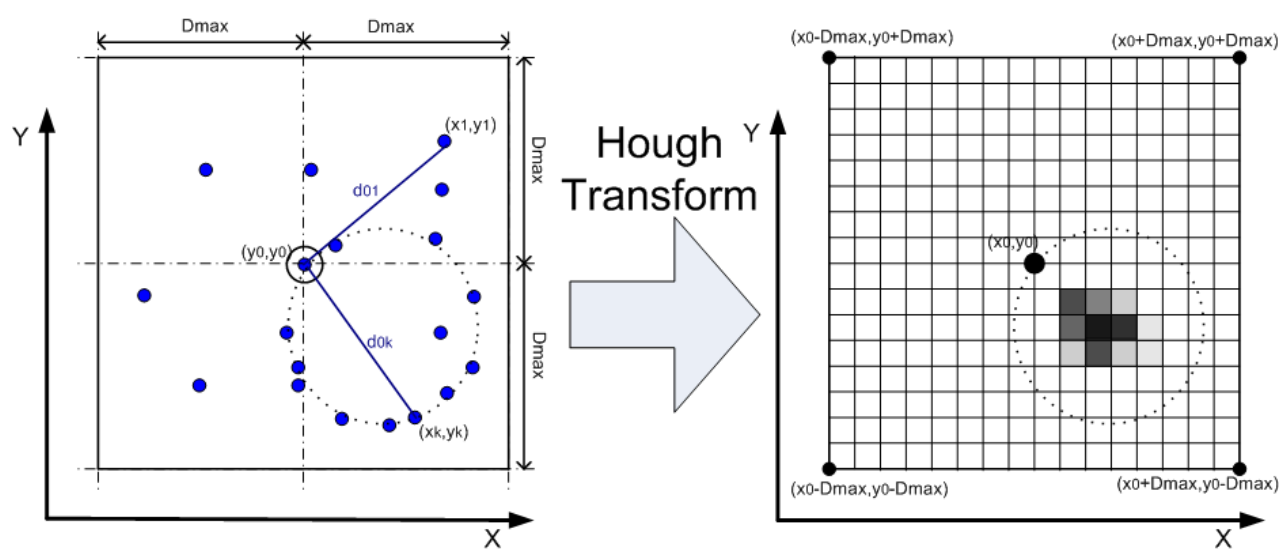

Figure 1: Preliminary local hit selection in a region defined by the maximum ring diameter plus a safety margin(left). Schematic view of the 2D histogram of ring centers (right).

Ring selection. The above described local ring-candidate search algorithm finds not only correct rings, but also wrong rings as a result of the high ring density in combination with noise hits. A set of ring characteristics had been investigated to be used for a quality estimation of found rings. After a statistical analysis six parameters were selected: the number of hits per ring; the three biggest angles between neighboring hits in the ring; the number of hits in a small corridor around the ring; the position of the ring in the RICH detector; radius, $\chi^{2}$ of the fit. An artificial neural network (ANN) was used for the ring quality calculation. The ANN derives the ring quality using the six input parameters described above. The selection of good rings from the array of found ring-candidates is based on the ring quality which has been calculated by the ANN. With this information, the algorithm compares the ring quality choosing good rings and rejecting repeatedly found rings (clones) and wrongly found rings. The algorithm checks for shared hits with all other ring-candidates. If the ring shares more than $25 \%$ of its hits with a better quality ring it is rejected.

Ring fitting. Both circle and ellipse fitting were implemented for the CBM RICH detector. Because of its simplicity and a very high computational speed circle fitting is used in the ring recognition algorithm. The algorithm which is known as COP (Chernov-Ososkov-Pratt) was implemented [5]. As the rings in the CBM RICH detector have a slight elliptic shape, an ellipse fitting algorithm based on the Taubin method [6] was implemented.

\section{Speedup of the algorithm}

Algorithm optimization. As the HT is the most time consuming part in the algorithm an optimization of the Hough Transform combinatorics is needed. A reduction of the combinatorics was done by dividing hits into several groups and performing the Hough Transform for each group independently and then summing up the obtained histograms in one. Other optimization includes: a precise prediction of the local area, mathematic optimization of the algorithm, memory usage optimization in order to store data in cache, removing the best fitted hits of found rings, algorithm parameter optimization etc.

SIMD and multithreading. SIMD is short for Single Instruction Multiple Data. It refers to a computing method that enables processing of multiple data with a single instruction. Requiring 
fewer instructions to process a given amount of data, SIMD operations yield higher computational speed than scalar operations. The Intel Streaming SIMD Extension (SSE) technology is supported by modern CPUs. Processors with Intel SSE support have a set of 128-bit registers, each of which may contain four 32-bit single-precision floating-point numbers. The best candidate for SIMDization is a computing intensive procedure without branches (if, break, continue), which can work in parallel for multiple data. Multi-core processors have made parallel programming more and more mainstream. Motivated by this fact the possibilities to use multithreading in the ring reconstruction were investigated. For this the Intel Threading Building Blocks (TBB) library was used [7]. TBB is a $\mathrm{C}++$ template library for parallelism. TBB supports scalable parallel programming, which means that programs using TBB will run on systems with a single processor core, as well as on systems with multiple processor cores.

Hough Transform and SIMD. The first step was to introduce float precision in the HT calculations instead of double precision. It was tested and our results showed that HT works without deterioration with float precision. Then the RICH data were vectorized. This means that each hit vector consists of 4 hit measurements: $X_{v}, Y_{v}$, where $X_{v}=\left(X_{0}, X_{1}, X_{2}, X_{3}\right) ; Y_{v}=\left(Y_{0}, Y_{1}, Y_{2}, Y_{3}\right)$. However $\mathrm{C}++$ code using SSE instructions uses different expressions compared to code with the corresponding scalar instructions. Rewriting the code using vector instructions would require to provide support for both, scalar and vector versions, duplicating modifications, debugging and testing. Therefore, the SSE vector instructions were set in a header file, overloading all operands and inlining several functions. In this way the source code remains the same, and possible changes of the code in the future will be valid for both, scalar and vector, versions. Using the SSE instructions the calculation of the ring parameters $(x, y, r)$ from 4 triplets at a time using the SIMD execution model was implemented.

Hough Transform and multithreading. Multithreading parallelization of the HT algorithm was done on different levels: 1) As the RICH detector consists of two independent photodetectors, the ring reconstruction was done in parallel for each photodetector; 2) the localized HT enables to use many independent, parallel local ring reconstructions; 3) Dividing hits into several groups during the triplet search and performing the HT for each group independently in parallel.

\section{Ring finding results}

In order to test the ring reconstruction algorithm, central $\mathrm{Au}+\mathrm{Au}$ collisions at $25 \mathrm{AGeV}$ beam energy were simulated with UrQMD [8]. Additional electrons at the primary vertex were embedded in these events in order to estimate their reconstruction efficiency. About 80 rings per event are seen in the RICH detector, mostly from secondary electrons. The comparison of the fast scalar version and the parallel version of the ring finding shows that the momentum integrated reconstruction efficiency is the same for both versions (93\%), see Figure 2. Overall, typically $4 \%$ of the approximately 80 found rings are fake rings and $1 \%$ clone rings.

A speed up of 62 is achieved by the optimization of the algorithm. Using SIMDization and multithreading the speed of the algorithm was increased by a factor of 2. In total a speed up factor of 119 was achieved (from $357 \mathrm{~ms} / \mathrm{event}$ to $3 \mathrm{~ms} / \mathrm{event}$ ) for the optimized parallel version in comparison to the initial algorithm (expected RICH event rate is around 25000 events per second). The lower than expected speedup factor of 2 for the parallelization is assumed to stem from the fact 

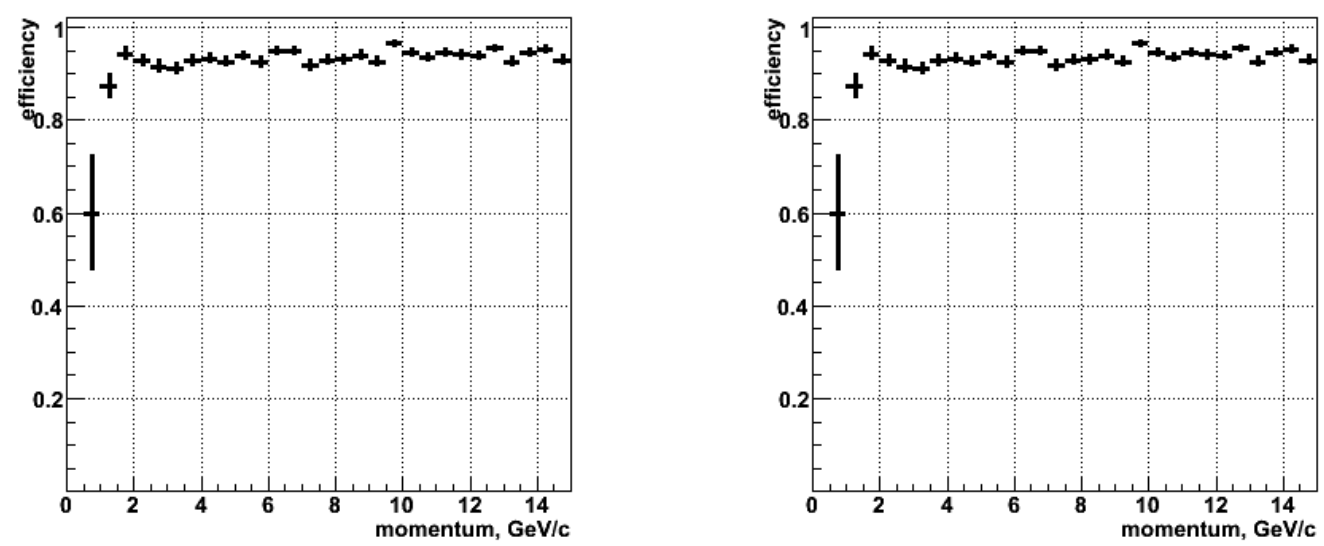

Figure 2: Ring finder efficiency for embedded electrons in dependence on momentum for central $\mathrm{Au}+\mathrm{Au}$ collisions at $25 \mathrm{AGeV}$ beam energy. Left: fast serial ring reconstruction algorithm. Right: parallel ring reconstruction algorithm.

that not all parts of the algorithm are parallelized yet and that there is too much time consumption in the synchronization between threads. Further investigations of the parallel ring reconstruction algorithm are ongoing.

\section{Summary}

Fast event reconstruction algorithms are essential for the CBM experiment. One of the challenge is ring recognition in the RICH detector. A fast and efficient algorithm for ring recognition in the CBM RICH was developed and improved considerably. A parallel version of the algorithm, which uses SIMD and multithreading was developed. The time for one event reconstruction in RICH (about 80 rings) is $5.8 \mathrm{~ms}$ for the optimized scalar version and $3 \mathrm{~ms}$ for the parallel version. Further investigations of the parallel version are ongoing.

\section{References}

[1] Compressed Baryonic Matter Experiment. Technical Status Report. 2005. http://www.gsi.de/documents/DOC-2005-Feb-447-1.pdf.

[2] C. Höhne et al. Nucl. Inst. and Meth. A 595, 187, 2008.

[3] S. Lebedev et al. "Ring Recognition and Electron Identification in the RICH detector of the CBM Experiment at FAIR", proceedings of the CHEP'09 conference, to be published in J. Phys.: Conf. Ser.

[4] P. V. C. Hough, Method and Means for Recognizing Complex Patterns, US Patent: 3, 069, 654, 1962.

[5] N. Chernov, G. Ososkov Comp. Phys. Comm., 33 p. 329-333, 1984.

[6] N. Chernov J Math Im Vi, 27 p. 231-239, 2007.

[7] Intel@ Threading Building Blocks. http: / / www . threadingbuildingblocks . org/.

[8] Bass S.A. et al. // Prog. Part. Nucl. Phys. V. 41. 1998. P. 255-370. 